\title{
Forschungsschwerpunkte in der Österreichischen Kinder- und Jugendpsychiatrie - 2007 bis 2017
}

\author{
Andreas Karwautz (D) - Cvetka F. Lipuš · Martin Fuchs
}

Eingegangen: 15. Mai 2017 / Angenommen: 13. Juni 2017 / Online publiziert: 30. August 2017

(c) Der/die Autor(en) 2017. Dieser Artikel ist eine Open-Access-Publikation.

Zusammenfassung Das „Sonderfach Kinder- und Jugendpsychiatrie" besteht seit 2007, 2015 wurde mit der Ausbildungsordnung 2015 eine Erweiterung definiert: „Sonderfach für Kinder- und Jugendpsychiatrie und Psychotherapeutische Medizin“. Um die Forschungsleistung der letzten 10 Jahre erstmals zu dokumentieren, wurden die in Pubmed sichtbaren Publikationen und Bücher der Mitglieder sowie die wissenschaftlich/klinischen Schwerpunkte der großen Ausbildungszentren in Österreich erfasst.

Die Mitglieder der ÖGKJP sind wissenschaftlich sehr aktiv und forschen zu verschiedenen Teilbereichen des Fachgebietes. Die meisten $(n=115)$ der 284 Publikationen (41\%) erschienen zu 5 Themen: Essstörungen, Forensik, Neuropädiatrie, Psychosen und Prodrome und Suizidalität. 13 der Arbeiten konnten aufgrund ihrer Qualität mit Preisen ausgezeichnet werden. Alle Abteilungen setzen - trotz Vollversorgungsauftrag mit zeitlich extrem hohem Anteil an

Zusatzmaterial online Zusätzliche Informationen sind in der Online-Version dieses Artikels (doi: 10.1007/s40211-0170234-1) enthalten.

Univ.-Prof. Dr. A. Karwautz, FAED ( $\varangle)$

Universitätsklinik für Kinder- und Jugendpsychiatrie,

Medizinische Universität Wien, Waehringer Guertel

18-20, 1090 Wien, Österreich

andreas.karwautz@meduniwien.ac.at

Mag. C. F. Lipuš, MLIS

Salzburger Universitätsklinikum, Paracelsus Medizinische Privatuniversität, Strubergasse 21, 5020 Salzburg, Österreich cvetka.lipus@pmu.ac.at

OA Dr. med. M. Fuchs

Universitätsklinik für Psychiatrie, Psychotherapie und Psychosomatik im Kindes- und Jugendalter, Medizinische Universität Innsbruck, Anichstraße 35, 6020 Innsbruck, Österreich

martin.fuchs@tirol-kliniken.at der Arbeitszeit - klinisch/wissenschaftliche Schwerpunkte und bieten vertiefende Versorgung in diesen speziellen Bereichen an. Ein weiterer Ausbau der Forschungskompetenzen sollte erfolgen und sollte auch gesundheitspolitisch priorisiert werden.

Schlüsselwörter Forschung - Kinder- und Jugendpsychiatrie - Bücher · PUBMED - Schwerpunkte

\section{Key-topics in Austrian Child and Adolescent Psychiatry 2007-2017}

Summary The medical speciality "Child and Adolescent Psychiatry" exists since 2007. 2015 a new training regulation defined an increment to "Child and Adolescent Psychiatry and Psychotherapeutic Medicine”. To document the achievements in research within the last 10 years for the first time, all papers listed in Pubmed and all books published by the member of the ÖGKJP were summarized. Additionally, the clinical/scientific key aspects of all large teaching centres were documented.

All members of the ÖGKJP are scientifically active in various areas of child psychiatry. Most of the 284 publications ( $n=115 ; 41 \%$ ) were published on 5 topics: eating disorders, forensic psychiatry, neuropediatrics, psychoses and prodromal states, suicidality. Thirteen of the papers have already been awared prices because of their high quality. All departments define clinical/scientific key areas - beside a time-consuming clinical work load - and offer specialized care. Further development of research competence should take place and should also have high priority in health politics.

Keywords Research - Child and Adolescent Psychiatry $\cdot$ Books $\cdot$ PUBMED $\cdot$ Key aspects 


\section{Einleitung}

Seit 2007 besteht das Sonderfach „Kinder- und Jugendpsychiatrie“ und seit 2016 die Erweiterung des Sonderfaches „Kinder- und Jugendpsychiatrie und Psychotherapeutische Medizin“. In der österreichischen Kinder- und Jugendpsychiatrie (KJP) werden an den bettenführenden Abteilungen, die in Österreich für Forschung und Ausbildung hauptverantwortlich zeichnen, verschiedene Krankheitsbilder im Rahmen von Spezialangeboten versorgt und beforscht. Ziel der Arbeit ist es, die Forschungsleistung der österreichischen Kinder- und Jugendpsychiatrie der letzten 10 Jahre erstmals möglichst umfassend quantitativ und in ihren Schwerpunkten zu erfassen. In ähnlicher und noch ausführlicher Form wurde von Hebebrand et al. [1] die Forschungslandschaft der deutschen Kinder- und Jugendpsychiatrie vermessen. Dort fanden sich als fünf wichtigste Themen die Forschung in den Bereichen „Körperliche Erkrankungen“, „Säuglings- und Kleinkindpsychiatrie“, „Forensik und Psychopathie“, „Schulische Entwicklungsstörung“ und „Grundlagenforschung“ [1].

\section{Preise für wissenschaftliche Arbeiten aus der ÖGKJP}

Da die Verleihung von Preisen für wissenschaftliche Arbeiten ein weiterer Indikator für Erfolg und eine positive Entwicklung eines Faches ist, freuen wir uns, dass zwischen 2008 und 201713 hervorragende wissenschaftliche Arbeiten, die für den „Wissenschaftspreis der ÖGKJP“ und den „Ernst Berger-Förderpreis für sozialpsychiatrische Forschung“ eingereicht wurden, prämiert werden konnten. Die Förderung des Ernst Berger Preises erfolgte 5 mal bis 2016 von E. Berger, ab 2017 durch das Kuratorium für psychosoziale Dienste in Wien. Die Themen und die Preisträger waren:

Wissenschaftspreis der ÖGKJP: 2017 Dr. Martin Fuchs: „Child and adolescent psychiatry patients coming of age: a retrospective longitudinal study of inpatient treatment in Tyrol“; 2016 Mag. Michael Zeiler: „Prevalence of Eating Disorders and Associations with Health-related Quality of Life“; 2015 Dr. Türkan Akkaya-Kalayci: „The impact of migration and culture on suicide attempts of children and adolescents living in Istanbul“; 2014 Dr. Mag. Maria Teresa Gutmann: „Psychische Gesundheit und Psychopathologie von Kindern und Jugendlichen“; 2013 Ass.-Prof. Mag. Dr. Gudrun Wagner: „Internet delivered cognitive behavioural therapy vs. conventional guided self-help in the treatment of bulimia nervosa: Long-term evaluation of a randomized controlled trial“; 2012 Mag. Monika Smetana „Die Wiederkehr des Ähnlichen Zur Bedeutung musikalischer Objekte in der Musiktherapie bei Jugendlichen mit strukturellen Störungen“ 2011 Assoc.-Prof. Dr. Julia Huemer: „Psychopathology in African unaccompanied refugee minors in Austria“.
2008 Dr. Christian Kienbacher: „Clinical features, classification and prognosis of migraine and tension-type headache in children and adolescents: a long-term follow-up study“.

Ernst Berger-Förderpreis für sozialpsychiatrische Forschung: 2017: Dr. Türkan Akkaya-Kalayci: „Psychiatric emergencies of minors with and without migration background“; 2016 Mag. Klemens Meister: „Einfluss externer Determinanten auf die gesundheitsbezogene Lebensqualität von Kindern und Jugendlichen mit psychischen Erkrankungen“; 2015 Mag. Dr. Julia Philipp: „The Mental Health in Austrian Teenagers (MHAT)-Study: preliminary results from a pilot study“; 2013 Mag. Petra Katzenschläger: „Lebenszufriedenheit und Symptomatik bei männlichen Jugendlichen mit niedriger Intelligenz und hoher psychosozialer Auffälligkeit im stationären rehabilitativen Setting“; 2012 Dr. Susanne M. Bauer: „Psychosocial background in incarcerated adolescents from Austria, Turkey and former Yugoslavia.“ 2009 Mag. Dr. Sandra Möstl: „Erziehungsbedürftig oder krank? Grenzfälle und Kooperationen zwischen stationärer Einrichtung der Jugendwohlfahrt und der Kinder- und Jugendpsychiatrie in der Steiermark."

\section{Methoden}

Um einen direkten und lückenlosen Einblick in die Leistungen $\mathrm{zu}$ erhalten wurden erstens primär die für Ausbildung und Forschung Hauptverantwortlichen, die Abteilungsleiter der 11 KJP-Primariate Österreichs (5 Universitätskliniken und 6 Landesabteilungen) angefragt, die klinisch/wissenschaftlichen Schwerpunkte ihrer Abteilung (seit 2007) kurz darzustellen. Auf die Anfrage bei den Leitern kleinerer Zentren (z. B. Tageskliniken und Ambulatorien), die Teilausbildungsberechtigung haben, wurde aus Praktikabilitätsgründen verzichtet. Es wurden zweitens alle Mitglieder der ÖGKJP per E-Mail angeschrieben, und um Bekanntgabe ihrer Buchveröffentlichungen ab 2007 (Herausgeberwerke oder Monographien herausgegeben oder verfasst von ÖGKJP-Mitgliedern) gebeten. Um einen möglichst umfassenden Überblick über die international sichtbaren Veröffentlichungen der österreichischen Kinder- und Jugendpsychiatrie zu bekommen, wurde drittens eine Pubmed-Recherche der Publikationen (zwischen 2007 und 2017) aller Mitglieder der ÖGKJP durch Fr. Mag. Lipuš, PMU Salzburg durchgeführt.

Da Bücher, die von Mitgliedern herausgegeben oder verfasst wurden, schwer umfassend bibliometrisch erfassbar sind, wurde eine direkte Mailanfrage (07.03.2017) an alle Mitglieder versendet. Information zu einzelnen Buchkapiteln wurden nicht abgefragt und konnten aus Platzgründen auch nicht berücksichtigt werden (z. B. [2]).

Die Pubmed-Recherche wurde gewählt, da die Publikationen mit internationaler Sichtbarkeit im Wesentlichen in dieser Datenbank aufscheinen. Die 


\section{schwerpunkt kinder- und jugendpsychiatrie}

Tab. 1 Die klinisch/wissenschaftlichen Schwerpunkte der 11 KJP Fachabteilungen Österreichs

\begin{tabular}{|c|c|c|c|c|}
\hline $\begin{array}{l}\text { Name der Abtei- } \\
\text { lung }\end{array}$ & Leiterln & $\begin{array}{l}\text { Wissenschaftlich/Klinische Schwerpunkte und deren } \\
\text { Leitung/Spezialangebote }\end{array}$ & $\begin{array}{l}\text { Ausrichtung von Fachtagungen und deren Lei- } \\
\text { tung }\end{array}$ & $\begin{array}{l}\text { Lehrbücher } \\
\text { (Zitat) }\end{array}$ \\
\hline \multicolumn{5}{|c|}{ Universitätskliniken } \\
\hline $\begin{array}{l}\text { UK Linz; } \\
\text { Standort Neuromed } \\
\text { Campus \& Standort } \\
\text { Med Campus IV }\end{array}$ & $\begin{array}{l}\text { Prim. Dr. } \\
\text { Michael } \\
\text { J. Merl } \\
\text { (2009-) }\end{array}$ & $\begin{array}{l}\text { Persönlichkeitsstörungen } \\
\text { J. Steininger } \\
\text { Trauma- und Traumafolgestörungen } \\
\text { K. Arthofer } \\
\text { ADHS } \\
\text { A. Demel, M. Merl } \\
\text { Jugendforensik } \\
\text { (im Aufbau; J. Steininger, M. Merl) } \\
\text { Jugendliche u. substanzgebundene Suchterkrankun- } \\
\text { gen } \\
\text { (im Aufbau; J. Steininger, Ch. Haas-Stockmair) } \\
\text { Essstörungen } \\
\text { M. Merl } \\
\text { Angst-/Zwangsstörungen } \\
\text { T. Preißler } \\
\text { Ticstörungen einschl. Tourette-Syndrom } \\
\text { T. Preißler }\end{array}$ & $\begin{array}{l}\text { Herbsttagung } 2009 \\
\text { „Das Therapeutische in der Kinder- u. Jugend- } \\
\text { psychiatrie - Interaktions-Chancen oder Split- } \\
\text { ter-Dasein“ W. Leixnering/S. Wiesinger } \\
10 \text { Jahre Jugendpsychiatrie } 2011 \\
\text { „Jetzt weiß ich, wer ich bin?! - } \\
\text { Therapiequalität in der Jugendpsychiatrie“ } \\
\text { W. Leixnering/S. Wiesinger } \\
\text { Österreichische Kinderschutzgruppen } 2014 \\
\text { „Psychische Gewalt und Kinderschutz" } \\
\text { M. Merl }\end{array}$ & - \\
\hline UK Innsbruck & $\begin{array}{l}\text { Univ.-Prof. } \\
\text { Dr. Kathrin } \\
\text { Sevecke } \\
(2013-)\end{array}$ & $\begin{array}{l}\text { Dimensionale Diagnostik der Persönlichkeitsstörungen } \\
\text { nach DSM-5 } \\
\text { (2013-) } \\
\text { K. Sevecke, A. Bock } \\
\text { Essstörungen } \\
\text { (2014-) } \\
\text { K. Sevecke, M. Gander, A. Bock } \\
\text { Pathologischer Internetgebrauch } \\
\text { (2014-) } \\
\text { M. Fuchs, K. Sevecke } \\
\text { Mentalisierungsbasierte Therapie } \\
\text { (2014-) } \\
\text { K. Sevecke, A. Bock, M. Reiter } \\
\text { Bindungsforschung } \\
\text { (2014-) } \\
\text { M. Gander, K. Sevecke } \\
\text { Neuropsychoimmunologie } \\
\text { (2014-) } \\
\text { K. Sevecke } \\
\text { Prädiktoren in der Adoleszenz für die Entwicklung } \\
\text { schwerer psychischer Störungen } \\
\text { (2007-) } \\
\text { A. Bock } \\
\text { Selbstverletzendes Verhalten } \\
\text { M. Fuchs, K. Sevecke, A. Bock } \\
\text { Operationalisierte Psychodynamische Diagnostik } \\
\text { A. Bock }\end{array}$ & $\begin{array}{l}\text { Innsbrucker Kinder- und Jugendpsychiatrie } \\
\text { Kongresse 2015, 2016, } 2017 \\
\text { K. Sevecke } \\
\text { Einführung in die Traumapädagogik } 2015 \\
\text { K. Sevecke } \\
\text { TFP Fortbildung } 2015 \\
\text { K. Sevecke } \\
\text { MBT Fortbildung } 2015 \\
\text { K. Sevecke } \\
\text { Suchtfortbildung } 2016 \\
\text { K. Sevecke } \\
\text { Fortbildung Psychische Auffälligkeiten \& Be- } \\
\text { handlungsmöglichkeiten bei Säuglingen und } \\
\text { Kindergartenkinder 2016 } \\
\text { K. Sevecke } \\
\text { TFP-A 2016 } \\
\text { K. Sevecke } \\
\text { Innsbrucker Tagung zu suchtbezogenen Störun- } \\
\text { gen im Jugendalter 2016 } \\
\text { K. Sevecke } \\
\text { Fortbildung TFP-A (2017-2019) } \\
\text { K. Sevecke } \\
\text { Forensik Curriculum (2017-2019) } \\
\text { K. Sevecke }\end{array}$ & {$[3,4]$} \\
\hline UK Salzburg & $\begin{array}{l}\text { Univ.-Prof. } \\
\text { Dr. Leonhard } \\
\text { Thun-Hohen- } \\
\text { stein }\end{array}$ & $\begin{array}{l}\text { Evaluation des stationären Behandlungskonzeptes } \\
\text { LTH, Corinna Fritz in Koop. mit LBI HTA Wien } \\
\text { Entwicklung eines Triagekonzeptes nach dem Man- } \\
\text { chestersystem } \\
\text { Belinda Plattner } \\
\text { Psychopathologie und Persönlichkeit „Ambulanzstu- } \\
\text { die“ } \\
\text { Belinda Plattner, Elisabeth Marte in Koop. m. UK f. KJP } \\
\text { Basel } \\
\text { Kinder- und Jugendforensik } \\
\text { Belinda Plattner, in Koop m. UK f KJP in Basel und } \\
\text { Zürich } \\
\text { Essstörungen, Epidemiologie, Behandlung } \\
\text { L. Thun-Hohenstein, J. Trost-Schrems } \\
\text { Synergetisches Navigationssystem } \\
\text { in Koop. mit Institut für Synergetik und Psychotherapie- } \\
\text { forschung der PMU Salzburg (Prof. Dr. G. Schiepek) }\end{array}$ & $\begin{array}{l}\text { Jahrestagungen der ÖGKJP 2007, } 2012 \text { \& } 2015 \\
\text { Politische Kindermedizin - jährlich } \\
\text { Symposium 5-Jahre Kinder- und Jugendpsychia- } \\
\text { trie Salzburg } 2014\end{array}$ & {$[5-10]$} \\
\hline
\end{tabular}


Tab. 1 (Fortsetzung)

\begin{tabular}{|c|c|c|c|c|}
\hline $\begin{array}{l}\text { Name der Abtei- } \\
\text { lung }\end{array}$ & Leiterln & $\begin{array}{l}\text { Wissenschaftlich/Klinische Schwerpunkte und deren } \\
\text { Leitung/Spezialangebote }\end{array}$ & $\begin{array}{l}\text { Ausrichtung von Fachtagungen und deren Lei- } \\
\text { tung }\end{array}$ & $\begin{array}{l}\text { Lehrbücher } \\
\text { (Zitat) }\end{array}$ \\
\hline UK Tulln & $\begin{array}{l}\text { Assoc.-Prof. } \\
\text { Prim. Dr. } \\
\text { Paulus Hoch- } \\
\text { gatterer }\end{array}$ & $\begin{array}{l}\text { Sprache, Narration und Selbstkonzepte } \\
\text { P. Hochgatterer } \\
\text { Institutionelle Erziehung und ihre Folgen } \\
\text { P. Hochgatterer } \\
\text { Transitionspsychiatrie } \\
\text { P. Hochgatterer, M. Aigner, Th. Meissel } \\
\text { Behandlung unter freiheitseinschränkenden Bedingun- } \\
\text { gen } \\
\text { P. Machowetz-Müllner } \\
\text { Störungen aus dem Autismusspektrum } \\
\text { P. Machowetz-Müllner } \\
\text { Traumafolgestörungen bei Kindern im Pflichtschulalter } \\
\text { U. Kogelbauer-Leichtfried } \\
\text { Schulvermeidende Störungsbilder } \\
\text { U. Kogelbauer-Leichtfried }\end{array}$ & $\begin{array}{l}\text { Jahrestagung der ÖGKJP (2009) } \\
\text { P. Hochgatterer, R. Fliedl, K. Ableidinger }\end{array}$ & - \\
\hline UK Wien & $\begin{array}{l}\text { Ass.-Prof. Dr } \\
\text { Christine Ve- } \\
\text { sely (interim.) } \\
\text { (2017-) } \\
\text { Prof } \\
\text { M. H. Fried- } \\
\text { rich (bis } \\
2013 \text { ), } \\
\text { Ass Prof } \\
\text { C. Vesely } \\
\text { (2013-2015), } \\
\text { Prof } \\
\text { L. Poustka } \\
\text { (2015-2016) }\end{array}$ & $\begin{array}{l}\text { ADHD } \\
\text { M. Abensperg-Traun, T. Akkaya-Kalayci Autismusspek- } \\
\text { trumstörungen (2015-) } \\
\text { L. Poustka } \\
\text { Essstörungen und Assoziierte Krankheitsbilder (2002-) } \\
\text { www.ess-stoerung.eu } \\
\text { A. Karwautz, G. Wagner } \\
\text { Forensik } \\
\text { S. Völkl-Kernstock } \\
\text { Kinder- und jugendpsychiatrische Epidemiologie } \\
\text { (2012-) } \\
\text { A. Karwautz, G. Wagner, M. Zeiler, J. Philipp } \\
\text { Neurofeedback (2016) } \\
\text { L. Konicar } \\
\text { Psychotherapie } \\
\text { S. Ohmann } \\
\text { Sucht } \\
\text { K. Skala } \\
\text { Therapeutisches Drug Monitoring in der KJP (2008-) } \\
\text { A. Karwautz, J. Huemer, M. Mitterer-Asadi } \\
\text { Transkulturelle Psychiatrie und migrationsbedingte } \\
\text { Störungen } \\
\text { T. Akkaya-Kalayci } \\
\text { Trauma } \\
\text { J. Huemer, S. Völkl-Kernstock, K. Skala } \\
\text { Kinderkopfschmerzen (1991-2015) } \\
\text { C. Wöber-Bingöl } \\
\text { Vorschulkinder (bis 2015) } \\
\text { C. Popow, S. Ohmann } \\
\text { Zwangsstörungen (bis 2015) } \\
\text { C. Popow, S. Ohmann }\end{array}$ & $\begin{array}{l}\text { Essstörungen und Assoziierte Krankheitsbilder } \\
\text { (jährlich seit 2009-) } \\
\text { A. Karwautz, G. Wagner, U. Bailer } \\
\text { Kinder- \& Jugendpsychiatrie (2010 \& 2013) } \\
\text { C. Kienbacher, A. Karwautz, B. Schuch, S. Völkl- } \\
\text { Kernstock, C. Vesely } \\
\text { Autismusspektrumstörungen (2015) } \\
\text { L. Poustka } \\
\text { Kopfschmerzen bei Kindern und Jugendlichen } \\
\text { (2008) } \\
\text { C. Wöber-Bingöl } \\
\text { Psychiatrie und Psychotherapie der Transition } \\
\text { (2016) } \\
\text { N. Kapusta, S. Bauer, R. Gößler, A. Karwautz, } \\
\text { N. Mossaheb, P. Plener, E. Pollak, C. Popow, } \\
\text { G. Psota, M. Schlögelhofer, K. Skala, H. E. Zesch }\end{array}$ & $\begin{array}{l}{[11-15,} \\
56]\end{array}$ \\
\hline \multicolumn{5}{|c|}{ Abteilungen an Landeskrankenanstalten } \\
\hline $\begin{array}{l}\text { Abteilung für Kin- } \\
\text { der- und Jugend- } \\
\text { psychiatrie und } \\
\text {-psychotherapie, } \\
\text { LKH Graz } \\
\text { Süd-West, Standort } \\
\text { Süd }\end{array}$ & $\begin{array}{l}\text { Prim. Dr. An- } \\
\text { na-Katharina } \\
\text { Purtscher- } \\
\text { Penz }\end{array}$ & $\begin{array}{l}\text { Psychosenachsorge } \\
\text { H. Andritsch } \\
\text { Suchtbezogene Störungen } \\
\text { M. Leitner, T. Trabi } \\
\text { Kinder und Jugendliche mit Migrations- und Fluchthin- } \\
\text { tergrund } \\
\text { T. Trabi, M. Pauger } \\
\text { Trauma und Krisenintervention } \\
\text { K. Purtscher-Penz }\end{array}$ & $\begin{array}{l}\text { Kinder- und Jugendpsychiatrisches Symposium } \\
\text { Pöllau (jährlich seit 2005) } \\
\text { K. Purtscher-Penz } \\
\text { Lehrgang Traumaädagogik in der Kinder- und } \\
\text { Jugendpsychiatrie und Jugendhilfe (2014) } \\
\text { K. Purtscher-Penz, C. Rothdeutsch-Granzer } \\
\text { Ausbildung Stressbewältigung nach belastenden } \\
\text { Ereignissen im Krankenhaus/Implementierung } \\
\text { am Standort Süd des LKH Süd-West (2010) } \\
\text { K. Purtscher-Penz, S. Monschein, W. Koller } \\
\text { Traumatherapie für Kinder und Jugendliche } \\
\text { (EMDR-Ausbildung) (2009) } \\
\text { K. Purtscher-Penz, T. Hensel } \\
\text { Lehrganges „Weiterbildung zur Fachkraft für } \\
\text { Kinder- und Jugendpsychiatrie“ (2009) } \\
\text { K. Purtscher-Penz }\end{array}$ & - \\
\hline
\end{tabular}


Tab. 1 (Fortsetzung)

\begin{tabular}{|c|c|c|c|c|}
\hline $\begin{array}{l}\text { Name der Abtei- } \\
\text { lung }\end{array}$ & Leiterln & $\begin{array}{l}\text { Wissenschaftlich/Klinische Schwerpunkte und deren } \\
\text { Leitung/Spezialangebote }\end{array}$ & $\begin{array}{l}\text { Ausrichtung von Fachtagungen und deren Lei- } \\
\text { tung }\end{array}$ & $\begin{array}{l}\text { Lehrbücher } \\
\text { (Zitat) }\end{array}$ \\
\hline $\begin{array}{l}\text { Kinder- und Ju- } \\
\text { gendpsychiatrie } \\
\text { und Psychiatrie } \\
\text { für behinderte } \\
\text { Erwachsene LHK } \\
\text { Rosenhügel }\end{array}$ & $\begin{array}{l}\text { Prim. Dr. } \\
\text { Ralf Gößler } \\
\text { (2007-) }\end{array}$ & $\begin{array}{l}\text { Früherkennung von Psychosen } \\
\text { R. Gößler } \\
\text { Entwicklungsambulanz } \\
\text { T. Elstner } \\
\text { Behindertenpsychiatrische Ambulanz } \\
\text { M. Langer } \\
\text { Ambulanz für somatoforme Störungen } \\
\text { A. Kreuzeder } \\
\text { Transitionspsychiatrie } \\
\text { R. Gößler } \\
\text { ADHS } \\
\text { R. Gößler } \\
\text { Psychoedukation bei Psychosen } \\
\text { R. Gößler, S. Ihra }\end{array}$ & $\begin{array}{l}\text { Psychiatrie und Psychotherapie der Transition } \\
\text { (2016) } \\
\text { N. Kapusta, S. Bauer, R. Gößler, A. Karwautz, } \\
\text { N. Mossaheb, P. Plener, E. Pollak, C. Popow, } \\
\text { G. Psota, M. Schlögelhofer, K. Skala, H. E. Zesch }\end{array}$ & - \\
\hline $\begin{array}{l}\text { Abteilung für Neu- } \\
\text { rologie und Psych- } \\
\text { iatrie des Kindes- } \\
\text { und Jugendalters, } \\
\text { Klinikum Klagenfurt }\end{array}$ & $\begin{array}{l}\text { Prim. Dr. } \\
\text { Wolfgang } \\
\text { Wladika } \\
\text { Prim. Doz. } \\
\text { G Spiel (bis } \\
\text { 2009) }\end{array}$ & $\begin{array}{l}\text { Mentalisationsbasierte Psychotherapie bei Störung des } \\
\text { Sozialverhaltens } \\
\text { W. Wladika } \\
\text { Arbeit und komplex und mehrfach Kranken } \\
\text { U. Lovsin Brunner } \\
\text { Interdisziplinäre Vernetzung } \\
\text { U. Lovsin Brunner } \\
\text { Eltern-Kind-Interaktionsdiagnostik und behandlung } \\
\text { A. Gasser } \\
\text { Suchtmedizin in der KJP } \\
\text { W. Wladika } \\
\text { Anorexie } \\
\text { E. Auer-Welsbach } \\
\text { Risiko- und Schutzfaktoren der frühen Kindheit } \\
\text { S. Pucker }\end{array}$ & $\begin{array}{l}\text { Kinder und Jugendpsychiatrie Jahrestagung } \\
\text { (2009) } \\
\text { G. Spiel } \\
\text { Lehrgang für Traumapädagogik (2015-2017) } \\
\text { W. Wladika }\end{array}$ & - \\
\hline $\begin{array}{l}\text { Abteilung für Kin- } \\
\text { der- \& Jugend- } \\
\text { psychiatrie und } \\
\text { Psychotherapie des } \\
\text { Landesklinikums } \\
\text { Mödling, Standort } \\
\text { Hinterbrühl }\end{array}$ & $\begin{array}{l}\text { Prim. Dr } \\
\text { Rainer Fliedl }\end{array}$ & $\begin{array}{l}\text { Operationalisierte Psychodynamische Diagnostik, } \\
\text { Schwerpunk Beziehungs- und Struktur-Achse } \\
\text { R. Fliedl, J. Noske, K. Zajec } \\
\text { Kooperation von Helfersystemen bei Kindern und } \\
\text { Jugendlichen } \\
\text { R. Fliedl, B. Winter, R. Zimmel } \\
\text { Gruppentherapie im stationären Setting } \\
\text { K. Zajec } \\
\text { Psychotherapie und Interpersonaler Raum } \\
\text { J. Noske } \\
\text { Evaluation von Behandlungserfolg und Behandlungszu- } \\
\text { friedenheit von therapeutischen Aufenthalten } \\
\text { P. Katzenschläger } \\
\text { Krisen und Deeskalation } \\
\text { R. Verbeck }\end{array}$ & $\begin{array}{l}\text { Hinterbrühler Symposien, alle } 2 \text { Jahre } \\
\text { http://www.inkiju.at/index.php/id-9- } \\
\text { hinterbruehler-symposium.html } \\
\text { (Fliedl, Bürger, Dühr) } \\
\text { Netzwerktagung Jährlich } \\
\text { http://www.kindernetzwerk.at/node/152 } \\
\text { (Plattform Kindernetzwerk) } \\
\text { Kinder und Jugendpsychotherapie } \\
\text { www.kijutagung.at/ } \\
\text { (Zajec, Fliedl) }\end{array}$ & [16-19] \\
\hline LKH Rankweil & $\begin{array}{l}\text { Prim. Maria } \\
\text { Veraar }\end{array}$ & Im Aufbau & - & - \\
\hline $\begin{array}{l}\text { 5. Psychiatrische } \\
\text { Abteilung für Kin- } \\
\text { der- und Jugend- } \\
\text { psychiatrie und } \\
\text { Psychotherapie } \\
\text { LKH Mauer Amstet- } \\
\text { ten }\end{array}$ & $\begin{array}{l}\text { Prim. Dr. } \\
\text { Karl Ablei- } \\
\text { dinger (seit } \\
\text { Gründung } \\
\text { 9/2008) }\end{array}$ & $\begin{array}{l}\text { Früherkennung und Diagnostik und Therapie von } \\
\text { „Grundstörungen“ } \\
\text { K. Ableidinger, D. Obereder } \\
\text { ADHS } \\
\text { K. Ableidinger, D. Obereder } \\
\text { Transitionspsychiatrie } \\
\text { M. Hartmann } \\
\text { Psychosomatische Störungen, insbesondere Anorexia } \\
\text { nervosa } \\
\text { K. Ableidinger, D. Hintsteiner, C. Sitz } \\
\text { Optimierung psychopharmakologischer Therapie } \\
\text { K. Ableidinger, C. Blach }\end{array}$ & $\begin{array}{l}\text { KJPP Mauer: Themenschwerpunkt: ADHS und } \\
\text { Teilleistungsstörungen (2008) } \\
\text { 10-Jahres-Feier der KJPP Mauer; The- } \\
\text { menschwerpunkt: Sinn und Spiritual Care im } \\
\text { Kinder- und Jugendalter (2018) }\end{array}$ & - \\
\hline
\end{tabular}

Namen aller Mitglieder der Mitgliederevidenz der ÖGKJP (Stand März 2017) wurden in die Recherche einbezogen. Die Abfrage erfolgte in der fortgeschrittenen Suche (,advanced search“) im Feldindex ,author". Alle Abfragen wurden auf den Zeitraum 2007 bis 2017 eingeschränkt. Weiteres wurde bei den Autoren/innen die Affiliation geprüft. In Zweifelsfällen wurde auch der Titel des Artikels und das Fachgebiet der Zeitschrift als ein weiteres Zuordnungskriterium herangezogen. Bei zu hoher (unplausibler) Anzahl an Treffern wurde eine weitere Eingrenzung mit dem Suchbegriff „Austria“ im Feld „affiliation“ durchgeführt. Im Falle von Doppelnamen wurden, falls keine Einträge im Autorenindex aufzufinden wa- 
ren, die Nachnamen auch separat abgefragt. Eine Einschränkung auf Originalarbeiten erfolgte nicht, als einschränkend wurde nur die Sichtbarkeit in Pubmed gewählt. Neben der Publikation in allen Journalen der Medizin wurden selbstverständlich auch die wichtigsten Zeitschriften des Fachgebietes (Zeitschrift für Kinder- und Jugendpsychiatrie, Journal of the American Academy of Child and Adolescent Psychiatry, Journal of Child Psychology and Psychiatry and Allied Disciplines, European Child and Adolescent Psychiatry, Development and Psychopathology, Child \& Adolescent Psychiatric Clinics of North America, Kindheit und Entwicklung, Child and Adolescent Mental Health, Child and Adolescent Psychiatry and Mental Health, Neuropsychiatrie, Praxis der Kinderpsychologie und Psychotherapie) eingeschlossen und in einer zusätzlichen Überprüfung auf etwaige durch die primäre Recherche nicht berücksichtigte Artikel hin kontrolliert. Lediglich die einschlägigen Zeitschriften „Kindheit und Entwicklung“, „Child and Adolescent Mental Health“ und „Pädiatrie und Pädologie“ sind im Web of Science, nicht aber in der Pubmed gelistet. Deren Erfassung ist nicht vollständig. Weitere Publikationen sind auf Nachfrage bei den Abteilungsleitern von diesen genannt worden, und wurden im Online Supplement Tab. 2 in der Publikationsliste aufgeführt.

\section{Ergebnisse}

\section{Die klinisch/wissenschaftlichen Schwerpunkte der 11 KJP Fachabteilungen Österreichs}

In der Tab. 1 werden die Schwerpunkte, Tagungen und Monographien, die aus den 11 Fachabteilungen zwischen 2007 und Februar 2017 hervorgegangen sind, dargestellt.

\section{Buchveröffentlichungen (Herausgeberwerke und Monographien)}

Die Bücher [3-19], die von den Mitgliedern genannt wurden, finden sich in der Tab. 1, dort den jeweiligen Abteilungen zugeordnet. Weitere Antworten gingen keine ein.

\section{Publikationen}

Die Mitglieder der ÖGKJP haben 264 in Pubmed gelisteten Arbeiten $\mathrm{zu}$ folgenden Themen publiziert (alphabetisch; Anzahl der Publikationen in Klammer): $\mathrm{Zu}$ manchen Themen wurde wenig/nichts publiziert. Verglichen mit dem Band zur Forschung in der DGKJP 2009 [19] waren ähnliche Schwerpunkte feststellbar, wobei eine tiefergehende Spezialisierung mit hohem wissenschaftlichem Output nur in einzelnen Abteilungen und für einige wenige Themen vorlag (z. B. Essstörungen, Kopfschmerzen, neuropädiatrische Erkrankungen, Autismusspektrumstörungen, Psychosentherapie, Affektive Störungen, Sucht, ADHD, Sui- zidologie, Transkulturelle Problematiken und Trauma am AKH Wien; Persönlichkeitsstörungen und Bindung an der MedUni Innsbruck; Forensik an der PMU Salzburg, etc.). Die universitären Abteilungen in Innsbruck, Tulln und Linz sind erst seit 2013 bzw. 2015 im Aufbau, die Abteilung in Rankweil ebenfalls.

In der 10-Jahres Periode wurden 264 in Pubmed gelistete Publikationen veröffentlicht, weitere 20 aus den oben erwähnten nicht gelisteten Journalen konnten zugefügt werden, sodass 284 Publikationen als Outcome der 10 Jahre KJP Österreich anzusehen sind. Die Vollständige Liste der Veröffentlichungen inkl. Abstracts erfolgt in einem Online-supplement.

\section{Online Supplement (link)}

Im Folgenden werden die Themen (alphabetisch) angeführt, zu denen von den Mitgliedern der ÖGKJP in diesem Zeitrahmen veröffentlicht wurde (in Klammer wird die Anzahl der Artikel genannt). Jedes Paper wurde nur einem Thema zugeordnet. ADHS (9), Adipositas (1); Angststörungen (2), Aus-, Fort- und Weiterbildung (4), Autismusspektrumstörungen (8), Bindung (2), Chronobiologie (1), Depression (7), Eltern-Kind Interaktion (5), Entwicklungsstörungen (1), Epidemiologie (6), Epilepsie (4), Essstörungen - inkl. Anorexia nervosa und Bulimia nervosa (34), Ethik (1), Forensik (23), Geschichte (2), Leitlinien (3), Methoden (7), Mediennutzung (1), Migration (2), Migräne (8), Minderjährige Flüchtlinge und Traumafolgestörungen (11), Neonatizid/Filizid (3), Neurobiologie/ Neuropsychologie (11), Neuropädiatrie - inkl. Medizin (22), Partizipation (1), Persönlichkeitsstörungen (10), Prävention (2), Psychopharmakologie (11), Psychosen und Prodrome (19), Psychosomatik (10), Psychotherapie (2), Public Health/Politik (2), Resilienz (3), Schmerz (1), Psychodynamik (5), Suchtstörungen - inkl. Alkohol (10), Suizidalität (17), Therapie (1), Temperament (1), Versorgungsforschung (4), Zwangsstörungen (1).

Die Mitglieder der ÖGKJP forschen zu verschiedenen Teilbereichen des Fachgebietes. Aus den Publikationen, die aus den 5 Schwerpunktthemen hervorgegangen sind werden 5 wichtige Publikationen ausgewählt, aus den weiteren 6 Themen 2. Die meisten der 284 Publikationen erscheinen zu 5 Themen: Essstörungen ( $n=34$ ) [20-24], gefolgt von Forensik ( $n=$ 23) [25-29], Neuropädiatrie $(n=22)$ [30-33], Psychosen und Prodrome $(n=19)$ [34-38] und Suizidalität ( $n=17$ ) [39-43]. Diese 5 Themen ergaben 115 Publikationen, was etwa $41 \%$ der Artikel ausmacht. Weitere sechs Themen, zu denen wenigstens 10 Arbeiten erschienen, sind Neurobiologie $(n=11)$ [44, 45], Minderjährige Flüchtlinge und Traumafolgestörungen ( $n=$ 11) [46, 47], Psychopharmakologie $(n=11)$ [48, 49], Persönlichkeitsstörungen $(n=10)$ [50, 51], Psychosomatik $(n=10)[52,53]$, Suchtstörungen $(n=10)[54,55]$ und ergaben 63 Artikel, was weitere $22 \%$ ausmacht. In Summe konzentrierte sich das wissenschaftliche Werk 
der ÖGKJP Mitglieder auf 11 Themen, die etwa $60 \%$ des Gesamtwerkes ausmachen. Die restlichen Publikationen waren auf 32 weitere Themen (1-9 Artikel) verteilt.

\section{Conclusio}

Die Mitglieder der ÖGKJP forschen zu verschiedenen Teilbereichen $(n=43)$ des Fachgebietes. Alle 11 KJP-Abteilungen in Österreich setzen - trotz Vollversorgungsauftrag mit zeitlich extrem hohem Anteil der klinischen Tätigkeit auch an universitären Einrichtungen - klinisch/wissenschaftliche Schwerpunkte und bieten vertiefende Versorgung in diesen speziellen Bereichen an. Ein weiterer Ausbau der Forschungskompetenzen sollte erfolgen und sollte auch gesundheitspolitisch priorisiert werden. Die Finanzierung von gemeinsamen Forschungsanstrengungen mit Vernetzung universitärer Forschungs-/ Versorgungsabteilungen und reiner Versorgungsabteilungen ist dringend anzuraten. Schwerpunktbildung mit dem Aufbau von Kompetenzzentren (wie sie schon bestehen z. B. an der MedUni Innsbruck für Persönlichkeitsstörungen, der PMU Salzburg für Forensik, der MedUniWien für Essstörungen und Neuropädiatrie) ist für die Teilnahme an internationalen Verbundstudien essentielle Voraussetzung. Die Weiterentwicklung von neuen und noch nicht im Zentrum stehenden Gebieten (z. B.: OPD-Forschung, epidemiologische [57] und Versorgungsforschung) ist neben den schon aktiven Hauptforschungsgebieten ebenfalls unbedingt förderungswürdig.

Open access funding provided by Medical University of Vienna.

Interessenkonflikt A. Karwautz, C.F. Lipuš und M. Fuchs geben an, dass kein Interessenkonflikt besteht.

Open Access Dieser Artikel wird unter der Creative Commons Namensnennung 4.0 International Lizenz (http:// creativecommons.org/licenses/by/4.0/deed.de) veröffentlicht, welche die Nutzung, Vervielfältigung, Bearbeitung, Verbreitung und Wiedergabe in jeglichem Medium und Format erlaubt, sofern Sie den/die ursprünglichen Autor(en) und die Quelle ordnungsgemäß nennen, einen Link zur Creative Commons Lizenz beifügen und angeben, ob Änderungen vorgenommen wurden.

\section{Literatur}

1. Hebebrand J, Albayrak Ö, Banaschewski T, Dittmann R, Fegert JM, Fendrich H, Föcker M, Freitag C, Gerlach M, von Gontard A, Häßler F, Herpertz-Dahlmann B, Hinney A, Klauck S, von Klitzing K, Konrad K, Laucht M, Moehler E, Poustka F, Ravens-Sieberer U, Resch F, Rothenberger A, Schimmelmann B, Schulte-Körne G, Schulte-Markwort M, Warnke A. Forschungsbericht. Forschungsleistung der deutschen Kinder- und Jugendpsychiatrie, Psychosomatik und Psychotherapie 2003-2008. Zeitschrift für Kinder- und Jugendpsychiatrie und Psychotherapie (2009), 37,pp. 231-367.DOI: 10.1024/1422-4917.37.4.231.
2. Lehmkuhl G, Poustka F, Holtmann M, Steiner H, der Lehrbuch KJP. Lehrbuch der Kinder- und Jugendpsychiatrie, Göttingen:Hogrefe; 2013.

3. Sevecke K, Krischer M. Persönlichkeitsstörungen im psychodynamischen Diskurs, Psychodynamik Kompakt, Vandenhoeck \& Ruprecht. 2016. ISBN 978-3525405598.

4. Bilke-Hentsch O, Sevecke K. Aggressivität, Impulsivität und Delinquenz von gesunden Aggressionen bis zur forensischen Psychiatrie bei Kindern und Jugendlichen. Stuttgart: Thieme; 2016. doi:10.1055/b-004-132246. ISBN 9783132038516.

5. KerblR, Thun-Hohenstein L, VavrikK, Waldhauser F. (Hrsg.) Kindermedizin - Werte versus Ökonomie. Wien, NewYork: Springer; 2007.

6. KerblR,Thun-HohensteinL, DammL, WaldhauserF. (Hrsg.) Kinder und Jugendliche im besten Gesundheitssystem der Welt. Wien, NewYork: Springer; 2010.

7. Thun-Hohenstein L. (Hrsg.) Kinder-und Jugendpsychiatrie in Österreich. Wien: KrammerVerlag; 2007.

8. Thun-Hohenstein L. (Hrsg.) Gewalt gegen Kinder und Jugendliche - Leitfaden für die Kinderschutzarbeit. BM f Gesundheit, Familie und Jugend. Wien: Ministeriumsbericht; 2008.

9. KerblR,Thun-HohensteinL,DammL, WaldhauserF.(Hrsg.) Kind und Recht. NewYork: Springer Verlag Wien; 2009.

10. Waldhauser F, Püspök R, Tatzer E, Thun-Hohenstein L. (Hrsg.) Das Kind zuerst - Probleme der Primärversorgung in Österreich. Wien: Springer; 2012.

11. Paulitsch K, Karwautz A. Grundlagen der Psychiatrie. WUV, UTBWien: Facultas; 2008.

12. Lebensraum Schule FMH. Perspektiven für die Zukunft. Wien: Ueberreuter; 2009.

13. Völkl-Kernstock S, Kienbacher C. (Hrsg.) Forensische. Psychologie-Medizin-Recht-Sozialarbeit. Springer Verlag: Arbeit mit Kindern und Jugendlichen. Praxishandbuch für die interdisziplinäreZusammenarbeit; 2016.

14. BergerE. NeuropsychologischeGrundlagenkindlicherEntwicklung. UTB. Wien: Böhlau;2010.

15. Berger E. (Hrsg.) Verfolgte Kindheit - Kinder und Jugendliche als Opfer der NS-Sozialverwaltung. Wien: Böhlau; 2007.

16. NoskeJ.(Hrsg.) SeelischeStrukturen:Versuch einerAbstimmung innerer und äußerer Wirklichkeiten in der jugendpsychiatrischen Behandlung. W: Krammer;2014.

17. Katzenschläger S, Gottwald T, Mannsberger T. Milieutherapie: Stationäre jugendpsychiatrische Betreuung unter Berücksichtigung des psychischen Strukturniveaus. W: Krammer; 2015.

18. VerbeekR.Aggression und das Deeskalieren bis in Grenzbereiche: Selbstwirksamkeit, Selbstbewusstsein und Grenzen im Umgang mitAggressionsdynamik. Independent, Publishing Platform: CreateSpace; 2015.

19. Schimak A. Über die Schamesröte einer jungen Turnusärztin. - Die Darstellung der Psychosomatik in Lehrbüchern der Medizin. Dr. Verlag: Kovac; 2014.

20. BoraskaV, Franklin CS, Floyd JA, Thornton LM, Huckins LM, Southam L, Rayner NW, Tachmazidou I, Klump KL, Treasure J, Lewis CM, Schmidt U, Tozzi F, Kiezebrink K, Hebebrand J, Gorwood P, Adan RA, Kas MJ, Favaro A, Santonastaso P, Fernandez-Aranda F, Gratacos M, Rybakowski F, DmitrzakWeglarz M, Kaprio J, Keski-Rahkonen A, Raevuori A, Van Furth EF, Slof-Op T, Landt MC, Hudson JI, Rechborn-Kjennerud T, Knudsen GP, Monteleone P, Kaplan AS, KarwautzA, Hakonarson H, Berrettini WH, GuoY, LiD, SchorkNJ, Komaki G, Ando T, Inoko H, Esko T, Fischer K, MannikK, Metspalu A, Baker JH, Cone RD, Dackor J, Desocio JE, Hilliard CE, O’Toole JK, Pantel J, Szatkiewicz JP, Taico C, Zerwas S, Trace 
SE, Davis OS, Helder S, Buhren K, Burghardt R, De Zwaan M, Egberts K, Ehrlich S, Herpertz-Dahlmann B, Herzog W, Imgart H, Scherag A, Scherag S, Zipfel S, Boni C, Ramoz N, Versini A, Brandys MK, Danner UN, De Kovel C, Hendriks J, Koeleman BP, Ophoff RA, Strengman E, Van Elburg AA, Bruson A, Clementi M, Degortes D, Forzan M, Tenconi E, Docampo E, Escaramis G, Jimenez-Murcia S, Lissowska J, Rajewski A, Szeszenia-Dabrowska N, Slopien A, Hauser J, Karhunen L, Meulenbelt I, Slagboom PE, Tortorella A, Maj M, et al. A genome-wide association study of anorexia nervosa. Mol Psychiatry. 2014;19:1085-94.

21. Karwautz AF, Wagner G, Waldherr K, Nader IW, FernandezAranda F, EstivillX, HollidayJ, Collier DA, Treasure JL. Geneenvironment interaction in anorexia nervosa: relevance of non-shared environment and the serotonin transporter gene. Mol Psychiatry. 2011;16:590-2.

22. Micali N, Holliday J, Karwautz A, Haidvogl M, Wagner G, Fernandez-Aranda F, Badia A, Gimenez L, Solano R, BreceljAnderlu M, Mohan R, Collier D, Treasure JL. Childhood eating and weight in eating disorders: a multi-centre European study of affected women and their unaffected sisters. Psychother Psychosom. 2007;76:234-41.

23. Wagner G, Penelo E, Wanner C, Gwinner P, Trofaier ML, Imgart $\mathrm{H}$, Waldherr $\mathrm{K}$, Woeber-Bingoel $\mathrm{C}$, Karwautz AF. Internet-delivered cognitive-behavioural therapy $\mathrm{v}$. conventional guided self-help for bulimia nervosa: long-term evaluation of a randomised controlled trial. Br J Psychiatry. 2013b;202:135-41.

24. Zeiler M, Waldherr K, Philipp J, Nitsch M, Dur W, Karwautz A, Wagner G. Prevalence of Eating Disorder Risk and Associations with Health-related Quality of Life: Results from a Large School-based Population Screening. Eur Eat Disord Rev. 2016;24:9-18.

25. Aebi M, Linhart S, Thun-Hohenstein L, Bessler C, Steinhausen HC, Plattner B. Detained Male Adolescent Offender's Emotional, Physical and Sexual Maltreatment Profiles and Their Associations to Psychiatric Disorders and Criminal Behaviors. JAbnorm Child Psychol. 2015;43:999-1009.

26. Mackey S, Chaarani B, Kan KJ, Spechler PA, Orr C, Banaschewski T, Barker G, Bokde AL, Bromberg U, Buchel C, Cattrell A, Conrod PJ, Desrivieres S, Flor H, Frouin V, Gallinat J, Gowland P, Heinz A, Ittermann B, Paillere Martinot ML, Artiges E, Nees F, Papadopoulos-Orfanos D, Poustka L, Smolka MN, Jurk S, Walter H, Whelan R, Schumann G, Althoff RR, Garavan H. Brain Regions Related to Impulsivity Mediate the Effects of Early Adversity on Antisocial Behavior. Biol Psychiatry.2016. doi:10.1016/j.biopsych.2015.12.027.

27. Plattner B, The SS, Kraemer HC, Williams RP, Bauer SM, Kindler J, Feucht M, Friedrich MH, Steiner H. Suicidality, psychopathology, and gender in incarcerated adolescents in Austria. J Clin Psychiatry. 2007;68:1593-600.

28. Steiner H, Erickson SJ, Maclean P, Medic S, Plattner B, Koopman C. Relationship between defenses, personality, and affect during a stress task in normal adolescents. Child PsychiatryHum Dev. 2007;38:107-19.

29. Voelkl-Kernstock S, Bein N, Gutschner D, Klicpera C, Ponocny-Seliger E, Friedrich MH. On the work of Austrian authorised experts on procedures in custodial and visiting rights a survey of current practice from the parents and children view. Neuropsychiatr. 2008;22:268-76.

30. Cury C, Toro R, Cohen F, Fischer C, Mhaya A, SamperGonzalez J, Hasboun D, Mangin JF, Banaschewski T, Bokde AL, Bromberg U, Buechel C, Cattrell A, Conrod P, Flor H, Gallinat J, Garavan H, Gowland P, Heinz A, Ittermann B, Lemaitre H, Martinot JL, Nees F, Paillere Martinot ML, Orfanos DP, Paus T, Poustka L, SmolkaMN, Walter H, Whelan R, Frouin V, Schumann G, Glaunes JA, Colliot O. Incomplete
Hippocampal Inversion: A Comprehensive MRI Study of Over 2000 Subjects. Front Neuroanat. 2015;9:160.

31. Freilinger M, Bebbington A, Lanator I, De Klerk N, Dunkler D, Seidl R, Leonard H, Ronen GM. Survival with Rett syndrome: comparing Rett's original sample with data from the Australian RettSyndrome Database. Dev Med Child Neurol. 2010;52:962-5.

32. Plecko B, Paul K, Paschke E, Stoeckler-Ipsiroglu S, Struys E, Jakobs C, Hartmann H, Luecke T, Di Capua M, Korenke C, Hikel C, Reutershahn E, Freilinger M, Baumeister F, Bosch F, Erwa W. Biochemical and molecular characterization of 18 patients with pyridoxine-dependent epilepsy and mutations of the antiquitin (ALDH7A1) gene. Hum Mutat. 2007;28:19-26.

33. Rabl U, Meyer BM, Diers K, Bartova L, Berger A, Mandorfer D, Popovic A, Scharinger C, Huemer J, Kalcher K, Pail G, Haslacher H, Perkmann T, Windischberger C, Brocke B, Sitte HH, Pollak DD, Dreher JC, Kasper S, PraschakRieder N, Moser E, Esterbauer H, Pezawas L. Additive geneenvironment effects on hippocampal structure in healthy humans. J Neurosci. 2014;34:9917-26.

34. Amminger GP, Schafer MR, Papageorgiou K, Klier CM, Cotton SM, Harrigan SM, Mackinnon A, Mc Gorry PD, Berger GE. Long-chain omega-3 fatty acids for indicated prevention of psychotic disorders: a randomized, placebocontrolled trial. Arch Gen Psychiatry. 2010;67:146-54.

35. Lancaster TM, Linden DE, Tansey KE, Banaschewski T, Bokde AL, Bromberg U, Buchel C, Cattrell A, Conrod PJ, Flor H, Frouin V, Gallinat J, Garavan H, Gowland P, Heinz A, Ittermann B, Martinot JL, Paillere Martinot ML, Artiges E, Lemaitre H, Nees F, Orfanos DP, Paus T, Poustka L, Smolka MN, Vetter NC, Jurk S, Mennigen E, Walter H, Whelan R, Schumann G. Polygenic Risk of Psychosis and Ventral Striatal Activation During Reward Processing in Healthy Adolescents. JAMAPsychiatry. 2016;73:852-61.

36. Lavoie S, Schafer MR, Whitford TJ, Benninger F, Feucht M, Klier CM, Yuen HP, Pantelis C, Mc Gorry PD, Amminger GP. Frontal delta power associated with negative symptoms in ultra-high risk individuals who transitioned to psychosis. Schizophr Res. 2012;138:206-11.

37. Mossaheb N, Schafer MR, Schloegelhofer M, Klier CM, Cotton SM, Mc Gorry PD, Amminger GP. Effect of omega-3 fatty acids for indicated prevention of young patients at risk for psychosis: when do they begin to be effective? Schizophr Res. 2013a;148:163-7.

38. Schafer MR, Klier CM, Papageorgiou K, Friedrich MH, Amminger GP. Early detection of psychotic disorders. Neuropsychiatr. 2007;21:37-44.

39. Aichhorn W, Fartacek R, Thun-Hohenstein L. Does antidepressant therapy increase suicide risk in children ad adolescents?-A comment. Neuropsychiatr. 2008a;22:16-22.

40. Dervic K, Brent DA, Oquendo MA. Completed suicide in childhood. Psychiatr Clin North Am. 2008;31:271-91.

41. Dervic K, Carballo JJ, Baca-Garcia E, Galfalvy HC, Mann JJ, Brent DA, Oquendo MA. Moral or religious objections to suicide may protect against suicidal behavior in bipolar disorder. JClin Psychiatry. 2011;72:1390-6.

42. Dervic K, Grunebaum MF, Burke AK, Mann JJ, Oquendo MA. Cluster C personality disorders in major depressive episodes: the relationship between hostility and suicidal behavior. Arch Suicide Res. 2007b;11:83-90.

43. Voracek M, Loibl LM, Kapusta ND, Niederkrotenthaler T, Dervic K, Sonneck G. Not carried away by a moonlight shadow: no evidence for associations between suicide occurrence and lunar phase among more than 65,000 suicide cases in Austria, 1970-2006. Wien Klin Wochenschr. 2008;120:343-9. 
44. Meyer BM, HuemerJ, Rabl U, Boubela RN, Kalcher K, Berger A, Banaschewski T, Barker G, Bokde A, Buchel C, Conrod P, Desrivieres S, Flor H, Frouin V, GallinatJ, Garavan H, HeinzA, Ittermann B, Jia T, Lathrop M, Martinot JL, Nees F, Rietschel M, Smolka MN, Bartova L, Popovic A, Scharinger C, SitteHH, Steiner H, Friedrich MH, Kaspar S, Perkmann T, PraschakRieder N, Haslacher H, Esterbauer H, Moser E, Schumann G, Pezawas L. Oppositional COMT Val158Met effects on resting state functional connectivity in adolescents and adults. Brain Struct Funct. 2016;221:103-14.

45. Mueller N, Baumeister S, Dziobek I, Banaschewski T, Poustka L. Validation of the Movie for the Assessment of Social Cognition in Adolescents with ASD: Fixation Duration and Pupil Dilation as Predictors of Performance. J Autism Dev Disord. 2016;46:2831-44.

46. Huemer J, Karnik N, Steiner H. Unaccompanied refugee children. Lancet. 2009a;373:612-4.

47. Voelkl-KernstockS, KarnikN, Mitterer-Asadi M, Granditsch E, Steiner H, Friedrich MH, Huemer J. Responses to conflict, family loss and flight: posttraumatic stress disorder among unaccompanied refugee minors from Africa. Neuropsychiatr. 2014;28:6-11.

48. Egberts K, Karwautz A, Plener PL, Mehler-Wex C, Kolch M, Dang SY, Taurines R, Romanos M, Gerlach M. Pharmacovigilance in child and adolescent psychiatry. Z Kinder Jugendpsychiatr Psychother. 2015;43:21-8.

49. ÖGKJP-Qualitätsssicherungkommission, Vesey C. 2013b. [Guideline for off-label use of psychotropic drugs in children and adolescents]. Neuropsychiatr, 27, 149-52.

50. Kosson DS, Neumann CS, Forth AE, Salekin RT, Hare RD, Krischer MK, Sevecke K. Psychopathy Checklist: Youth Version (PCL:YV) in adolescent females. Psychol. Factor structure of the. Bd.25.2013, S. 71-83.
51. Sevecke SK, Schmeck K, Krischer M. The dimensionalcategorical hybrid model of personality disorders in DSM5 from an adolescent psychiatric perspective - criticism and critical outlook. Z Kinder Jugendpsychiatr Psychother. 2014;42:279-83.

52. Wagner G, Zeiler M, Berger G, Huber WD, Favaro A, Santonastaso P, KarwautzA. Eating Disorders in Adolescents with Celiac Disease: Influence of Personality Characteristics and Coping. Eur Eat Disord Rev. 2015b;23:361-70.

53. Wagner G, Zeiler M, Grylli V, Berger G, Huber WD, Woeber C, Rhind C, KarwautzA. Coeliac disease in adolescence: Copingstrategies and personality factorsaffecting compliance with gluten-free diet. Appetite. 2016;101(54):55-61.

54. French L, Gray C, Leonard G, Perron M, Pike GB, Richer L, Seguin JR, Veillette S, Evans CJ, Artiges E, Banaschewski T, Bokde AW, Bromberg U, Bruehl R, Buchel C, Cattrell A, Conrod PJ, Flor H, Frouin V, Gallinat J, Garavan H, Gowland P, Heinz A, Lemaitre H, Nartinot JL, Nees F, Orfanos DP, Pangelinan MM, Poustka L, Rietschel M, Smolka MN, Walter H, Whelan R, Timpson NJ, Schumann G, Smith GD, Pausova Z, Paus T. Early Cannabis Use, Polygenic Risk Score for Schizophrenia and Brain Maturation in Adolescence. JAMA Psychiatry. 2015;72:1002-11.

55. Skala K, Reichl L, Ilias W, Likar R, Grogl-Aringer G, Wallner C, Schlaff G, Herrmann P, Lesch O, Walter H. Can we predict addiction to opioid analgesics? A possible tool to estimate the risk of opioid addiction in patients with pain. Pain Physician. 2013;16:593-601.

56. LehrnerJ, Stolba K, Traun-Vogt G, Völkl-KernstockS. (Hrsg.) Klinische Psychologie im Krankenhaus. Wien, New York: Springer; 2011.

57. Wagner G, Zeiler M, Waldherr K, Philipp J, Truttmann S, Dür W, Treasure JL, Karwautz AFK. DSM-5 criteria. Eur Child Adolesc Psychiatry. 2017. doi:10.1007/s00787-017-0999-6. 